\title{
Effect of Antazoline on Electrophysiological Properties of Atrial Muscle and Conduction System of the Heart
}

\author{
Bartłomiej Jacek Bińkowski ${ }^{1} \cdot$ Marcin Makowski ${ }^{1}$ Paweł Kubiński ${ }^{1} \cdot$ Andrzej Lubiński $^{1}$
}

Published online: 6 April 2018

(C) The Author(s) 2018

\begin{abstract}
Purpose Antazoline is a first-generation antihistaminic agent with additional anticholinergic properties and antiarrhythmic potential. Recent data shows its high effectiveness in sinus rhythm restoration among patients with paroxysmal atrial fibrillation. The effect of antazoline on electrophysiological parameters of the heart in vivo has not yet been examined. The aim of this study was to evaluate changes in electrophysiological parameters of the heart muscle and conduction system as a response to increasing doses of antazoline.

Methods After successful ablation of supraventricular arrhythmias, the electrophysiological parameters: sinus rhythm cycle length (SRCL), AH, HV, QRS, QT, QTc intervals, Wenckebach point (WP), sinus node recovery period (SNRT), intra- (hRACSos) and interatrial conduction time (hRA-CSd), right and left atrium refractory period (RA-; LA-ERP), and atrioventricular node refractory period (AVN-ERP) were assessed initially and after 100, 200, and $300 \mathrm{mg}$ of antazoline given intravenously.

Results Fifteen patients (8 males, 19-72 years old) undergoing EPS and RF ablation were enrolled. After $100 \mathrm{mg}$ bolus, a significant reduction in SRCL was noticed. After antazoline administration, significant prolongation of HV, QRS, QTc, hRACSos, hRA-CSd intervals, RA- and LA-ERP and reduction of SRCL were observed. After a total dose of $300 \mathrm{mg}$, QT interval prolonged significantly. Increasing the dose of antazoline had no impact on AH, Wenckebach point, AVN-ERP, and SNRT.

Conclusion Antazoline has an effect on electrophysiological parameters of the atrial muscle and has rapid onset of action. No negative effect on sinus node function and atrioventricular conduction in a unique property among antiarrhythmic drugs.
\end{abstract}

Keywords Antazoline $\cdot$ Antiarrhythmic drug $\cdot$ Electrophysiological study

\section{Introduction}

Antazoline is a first-generation antihistaminic agent with additional anticholinergic properties, first described in 1947 [1]. Its antiarrhythmic potential was first discovered in the 1950s of the last century [2-4]. In subsequent years, the effects of antazoline administered both orally and intravenously on different types of arrhythmias were assessed. There were antiarrhythmic effects against premature atrial and ventricular beats, nodal reentrant tachycardia and atrial and ventricular tachycardia. Initially, poor effect was noticed against atrial fibrillation (AF), probably

Bartłomiej Jacek Bińkowski

bartekbinkowski@gmail.com

1 Department of Invasive Cardiology and Cardiac Arrhythmias, University Clinical Hospital Military Memorial Medical Academy Central Veterans' Hospital in Lodz, Żeromskiego 113, 90-549 Łódź, Poland because the great part of sinus rhythm restoration attempts was made among patients with longstanding persistent arrhythmia [5-8]. Only one paper described 66\% success rate in AF treatment [9]. Moreover, complications from oral use of antazoline, mainly immunological and hematological, caused its widespread chronic use as an antiarrhythmic agent to be abandoned [10]. In 2000, new data on the efficacy of antazoline was published. Retrospective analysis of 1325 patients treated with intravenous antazoline for paroxysmal AF revealed that antazoline restored sinus rhythm in $52 \%$ of the patients [11]. Recent data indicate an even higher success rate approaching $80 \%$. The superiority of the antazoline over placebo in sinus rhythm restoration in recent onset AF has been confirmed in the AnPAF study. Complications of ad hoc administered antazoline seem to be rare and benign [12-15]. The effect of antazoline on electrophysiological parameters of the heart in vivo has not yet been examined. Thus, the aim of this study was to evaluate changes in electrophysiological parameters of the heart muscle and conduction system as a response to increasing doses of antazoline. 


\section{Methods}

\section{Patient Population}

After obtaining approval from the Ethics Committee (RNN/ 584/11/KB; 12.07.2011), we enrolled patients undergoing electrophysiological study for paroxysmal arrhythmias: atrioventricular nodal reentrant tachycardia (AVNRT), atrioventricular reentrant tachycardia (AVRT), and common atrial flutter (AFL). The main inclusion criteria were successful radiofrequency (RF) ablation of underlying arrhythmia and stable therapeutic effect of the ablation lasting for at least $15 \mathrm{~min}$ since last RF application. We included patients over the age of 18 without concomitant disease. Patients were off the antiarrhythmic drugs for at least five drug half-lives. Exclusion criteria were abnormal echocardiography findings (chambers dimensions, systolic and diastolic function of the ventricles, greater than mild valve disorders), use of amiodarone within last 3 months, use of drugs affecting QT interval, abnormal lab tests (full blood count, GFR, plasma electrolytes, ALT), need for use of drugs others than unfractionated heparin during the ablation (isoproterenol, atropine, ephedrine, antiarrhythmics, etc.), or recurrence of underlying arrhythmia. Written informed consent was obtained from each patient before entry into the study.

\section{Electrophysiological Study}

For electrophysiological study (EPS), three catheters were introduced through the right femoral vein and placed in high right atrium (hRA), coronary sinus (CS), and His bundle region (HIS). EPS evaluated the sinus rhythm cycle length (SRCL) averaged from 10 consecutive cycles, duration of $\mathrm{AH}, \mathrm{HV}$, QRS, QT, QTc (Bazett formula) intervals taken on sinus rhythm, Wenckebach point (WP), sinus node recovery period (SNRT), intra- and inter atrial conduction times measured as hRA- CS ostium interval (hRA-CSos) and hRA-CS distal interval (hRACSd), right and left atrium refractory period (RA-ERP, LAERP), and atrioventricular node refractory period (AVN-ERP). After initial measurements, antazoline was administered in $100 \mathrm{mg} / 3 \mathrm{~min}$-boluses up to a total dose of $300 \mathrm{mg}$. The dose and administration rate were based on doses recommended for arrhythmia treatment in Summary of Product Characteristics. Five minutes after initiation of every antazoline bolus, all of the measurements were repeated, except SNRT which was taken initially and after the full dose of antazoline. The time interval between the beginning of each injection was $10 \mathrm{~min}$. During the entire EPS, blood pressure and saturation were monitored noninvasively. After the study, ECG was monitored for at least $2 \mathrm{~h}$.

\section{Statistical Analysis}

The Shapiro-Wilk test was used to assess distribution of data. Data is shown as mean and standard error of the mean (SEM).
Statistical analysis was performed using non-parametric statistics. Wilcoxon matched pair signed-rank test was used to compare two time points. Friedman's and Dunn's test was used to compare four points in the same group. Statistical significance was defined as $p<0.05$.

\section{Results}

We enrolled 15 patients (8 males, 19-72 years old, EF $61 \pm$ $2.5 \%)$ undergoing EPS and RF ablation. The indications for EPS were AVNRT $(n=6)$, AVRT $(n=4)$, and AFL $(n=5)$. After $100 \mathrm{mg}$ bolus, a significant reduction in SRCL and QTc prolongation was noticed. After a total dose of $200 \mathrm{mg}$, we observed significant prolongation of $\mathrm{HV}, \mathrm{QRS}, \mathrm{hRA}-\mathrm{CS}$ s and hRA-CSd intervals and RA- and LA-ERP. After a total dose of $300 \mathrm{mg}$, QT interval prolonged significantly. Increasing the dose of antazoline had no impact on AH, WP, AVN-ERP, and SNRT. The results are summarized in Table 1 and on Fig. 1. In one patient, after total dose of $300 \mathrm{mg}$ of antazoline, we induced common atrial flutter with incremental atrial pacing. Arrhythmia cycle length was $200 \mathrm{~ms}$ with 2:1 atrioventricular conduction. The basic EPS finding in this patient was AVNRT and there was no evidence of pre-existing common atrial flutter. Arrhythmia was terminated with overdrive atrial pacing. After full dose of antazoline, two patients reported hot flush sensation and one nausea that resolved without any intervention.

\section{Discussion}

To our knowledge, this is the first study assessing effect of antazoline on electrophysiological parameters of the human heart. The data on antazoline effectiveness in ad hoc AF termination is constantly growing. After several nonrandomized observational studies the result of randomized clinical trial comparing antazoline and placebo for sinus rhythm restoration in paroxysmal AF has been published showing high effectiveness in sinus rhythm restoration exceeding $70 \%$. The rate of spontaneous sinus rhythm recoveries in placebo arm was only $10 \%$ [15]. While chronic antiarrhythmic therapy is practically impossible due to serious complications, it is still a very attractive option for rapid $\mathrm{AF}$ termination and in some countries it is registered and widespread used for this purpose.

Our results clearly indicate influence of antazoline on heart conduction system and muscle. Measured parameter changes: HV prolongation, QRS widening, atrial refractory period prolongation, and conduction velocity slowing are similar to agents classified as class I of Vaughan Williams classification of antiarrhythmic drugs. The constellation of changes of parameters in EPS suggests an influence of antazoline mainly on sodium channels. Moreover, known anticholinergic properties 
Table 1 Results summary. SRCL sinus rhythm cycle length, $h R A-C S o s$ intraatrial conduction time, $h R A-C S d$ interatrial conducion time, $R A-E R P$ right atrium refractory period, $L A-E R P$ left atrium refractory, $A V N-E R P$ atrioventricular node refractory period, WP Wenckebach point, $S N R T$ sinus node recovery period, BPsys systolic blood pressure, BPdias diastolic blood pressure

\begin{tabular}{|c|c|c|c|c|c|c|c|}
\hline & Baseline & $100 \mathrm{mg}$ & $P$ & $200 \mathrm{mg}$ & $P$ & $300 \mathrm{mg}$ & $P$ \\
\hline SRCL (ms) & $772.8 \pm 38.47$ & $703.4 \pm 32.51$ & $<0.01$ & $703.5 \pm 31.03$ & $<0.05$ & $702.9 \pm 31.31$ & $<0.01$ \\
\hline $\mathrm{AH}(\mathrm{ms})$ & $90.26 \pm 5.28$ & $93.93 \pm 6.47$ & $\mathrm{~ns}$ & $96.06 \pm 6.34$ & $\mathrm{~ns}$ & $97.13 \pm 5.59$ & $\mathrm{~ns}$ \\
\hline HV (ms) & $49.3 \pm 2.22$ & $54 \pm 3.09$ & ns & $56.46 \pm 2.89$ & $<0.001$ & $58.53 \pm 2.89$ & $<0.001$ \\
\hline QRS (ms) & $91.93 \pm 4.84$ & $95.13 \pm 5.64$ & ns & $99.27 \pm 6.43$ & $<0.01$ & $102.73 \pm 6.21$ & $<0.001$ \\
\hline QT (ms) & $356 \pm 7.54$ & $363.33 \pm 7.87$ & $\mathrm{~ns}$ & $363.33 \pm 7.29$ & $\mathrm{~ns}$ & $369.13 \pm 7.20$ & $<0.001$ \\
\hline QTc (ms) & $408.07 \pm 38.18$ & $434.67 \pm 9.89$ & $<0.05$ & $435.73 \pm 20.51$ & $<0.01$ & $443 \pm 3.53$ & $<0.001$ \\
\hline hRA-Csos (ms) & $83.2 \pm 5.39$ & $87.87 \pm 5.68$ & $\mathrm{~ns}$ & $91.4 \pm 5.1$ & $<0.01$ & $93.13 \pm 5.67$ & $<0.001$ \\
\hline hRA-CSd (ms) & $117.13 \pm 6.75$ & $122.8 \pm 7.13$ & ns & $126.67 \pm 6.64$ & $<0.001$ & $129.73 \pm 7.15$ & $<0.001$ \\
\hline AVN-ERP (ms) & $290.67 \pm 20.15$ & $298.67 \pm 18.97$ & ns & $278 \pm 18.24$ & $\mathrm{~ns}$ & $252 \pm 22.39$ & $\mathrm{~ns}$ \\
\hline RA-ERP (ms) & $210.67 \pm 7.27$ & $232 \pm 6.77$ & ns & $239.33 \pm 8.31$ & $<0.05$ & $246.67 \pm 8.71$ & $<0.001$ \\
\hline LA-ERP (ms) & $230.67 \pm 4.52$ & $236.67 \pm 4.65$ & ns & $243.33 \pm 4.75$ & $<0.05$ & $246 \pm 5.84$ & $<0.01$ \\
\hline WP (bpm) & $169.53 \pm 6.99$ & $168.73 \pm 7.49$ & ns & $168.2 \pm 7.71$ & $\mathrm{~ns}$ & $168.2 \pm 7.63$ & ns \\
\hline SNRT (ms) & $924.2 \pm 47.55$ & N/A & & N/A & & $899.53 \pm 40.60$ & ns \\
\hline BPsys (mmHg) & $123.46 \pm 15.55$ & N/A & & N/A & & $120.47 \pm 13.43$ & ns \\
\hline BPdias (mmHg) & $71.33 \pm 17.67$ & N/A & & N/A & & $73 \pm 15.55$ & $\mathrm{~ns}$ \\
\hline
\end{tabular}

N/A not available, $n s$ not significant

place antazoline near class Ia agent: quinidine. This data is consistent with the ELEPHANT study results. The early effect on surface ECG is similar to sodium channel blocker. In the ELEPHANT study, observation time was longer and revealed potential potassium channels inhibition and more pronounced QT interval prolongation in the later phase of observation [16]. In our group observation, period was probably too short to disclose these changes, but QT prolongation was also noticed.

QTc prolongation is very important finding of our study. QTc change resulted mainly from rhythm acceleration without simultaneous absolute QT interval shortening. Many patients with paroxysmal AF are treated for rhythm control with medications prolonging QT interval, mainly class III antiarrhythmic drugs. It is necessary to keep caution in these patients or consider abandoning use of antazoline for sinus rhythm restoration.

Our observations confirm another important property of antazoline: rapid onset of action. We noticed significant influence on electrophysiological parameters in measurements obtained after a total dose of $200 \mathrm{mg}$ and $20 \mathrm{~min}$ from the beginning of first bolus. Shortening of SRCL appeared almost immediately, after first bolus, and remained at the same level after further doses. This effect apparently results from rapid anticholinergic action of antazoline. Clinical data showed short time to AF cessation during WPW ablation. From the beginning of antazoline infusion, it took on average $7 \mathrm{~min}$ to restore sinus rhythm ${ }^{12}$. In the AnPAF study, mean time to sinus rhythm restoration was only 16 min [15]. This period could have been shorter than that required to significantly change the electrophysiological properties of the atria in our observation and only the SRCL has changed this early; however, the antazoline administration protocol differed between the studies. Thus, inhibition of parasympathetic ganglia may play an important role in quick AF cessation. Among antiarrhythmic drugs, only the recently approved vernakalant is comparably fast in sinus rhythm restoration $[17,18]$. Other commonly used agents usually require hours to achieve AF termination.

One of the most common side effects of antiarrhythmic therapy is bradycardia related to sinus node dysfunction or conduction disturbances. We did not notice any negative effects of antazoline either on sinus node function nor atrioventricular conduction. It has even accelerated sinus rhythm. Thanks to such properties, it may potentially become the drug of choice in patients with tachycardia - bradycardia syndrome, diagnosed sinus node dysfunction, or impaired atrioventricular conduction not protected with permanent pacemaker.

Our observations, together with previous studies on antazoline efficacy, build a basis for large scale, randomized trials on antazoline as a drug of choice for rapid, ad hoc treatment of recent onset AF.

\section{Study Limitations}

Patients with paroxysmal arrhythmias and after arrhythmia induction in EP study may response differently to antazoline in comparison to healthy people. Study involving healthy volunteers would be impossible to conduct due to ethical issues. Plasma drug levels were not assessed in the study, thus, association between electrophysiological parameters and drug concentration is unknown. 
Fig. 1 Effect of antazoline on electrophysiological properties of atrial muscle and conduction system of the heart. SRCL, sinus rhythm cycle length; hRA-CSos, intraatrial conduction time; hRACSd interatrial conducion time; RA-ERP right atrium refractory period; LA-ERP, left atrium refractory; AVN-ERP,

atrioventricular node refractory period; WP, Wenckebach point; SNRT, sinus node recovery period; T0 initial value, T100 after 100, T200 after 200, T300 after $300 \mathrm{mg}$ of antazoline. $* p<0.05, * * p<0.01$, $* * * p<0.001$ a

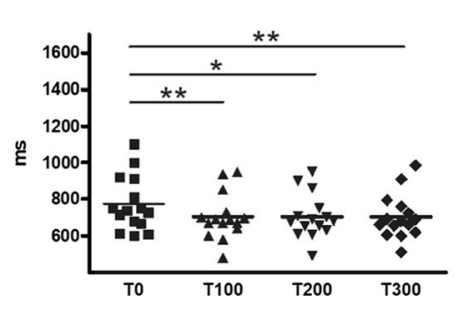

C

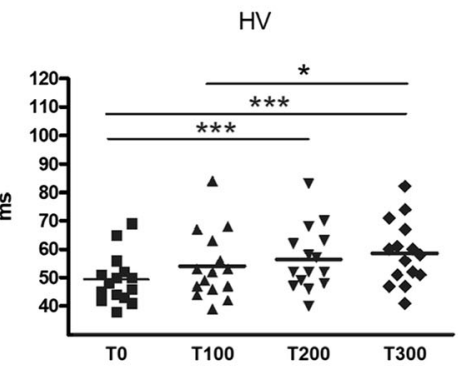

e

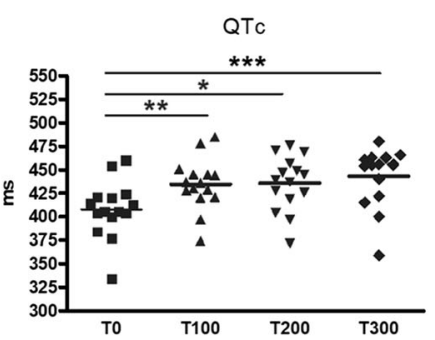

g

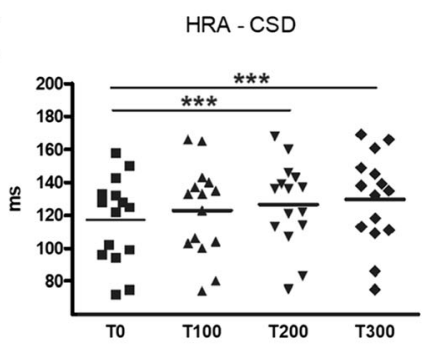

i

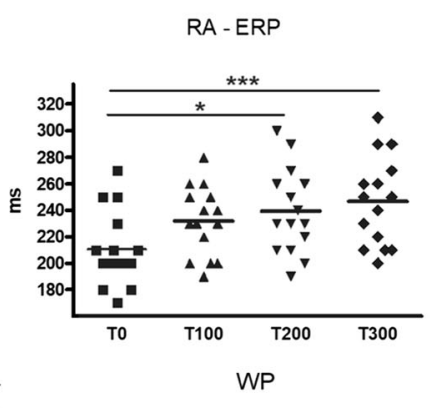

k

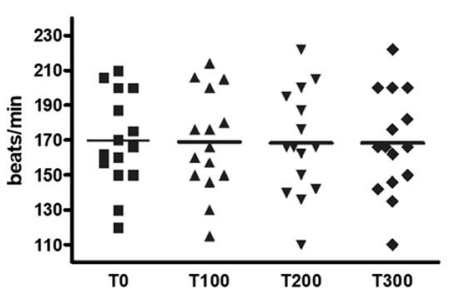

b

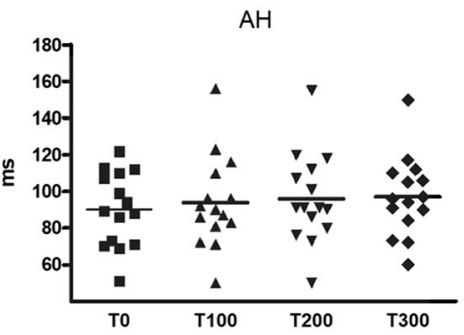

d

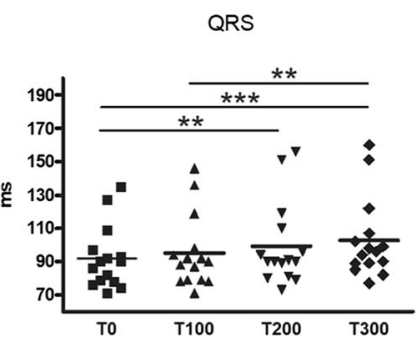

f

hRA - CSos

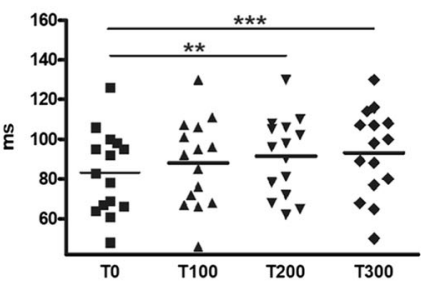

h

AVN - ERP

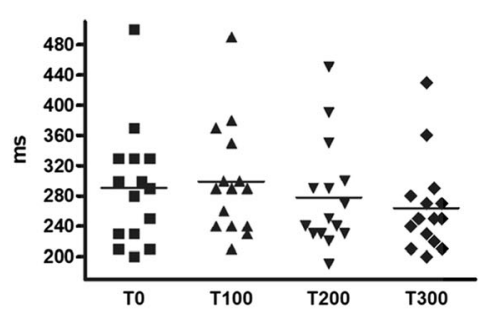

LA - ERP
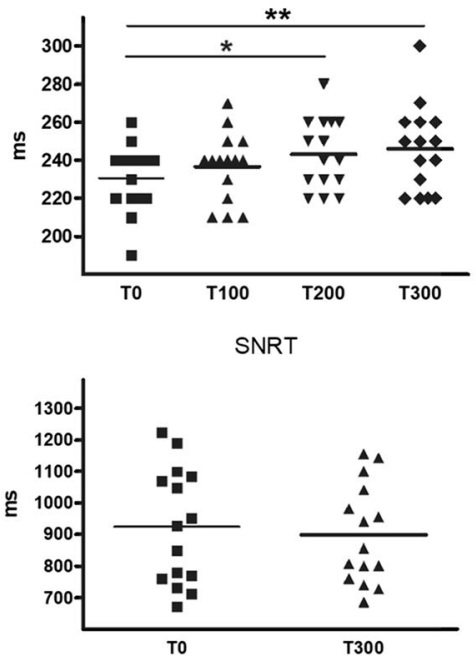


\section{Conclusion}

Antazoline has an effect on electrophysiological parameters of the atrial muscle and has rapid onset of action. No negative effect on sinus node function and atrioventricular conduction in a unique property among antiarrhythmic drugs.

\section{Compliance with Ethical Standards}

Conflict of interest The authors declare that they have no conflict of interest.

Ethical Approval All procedures performed in studies involving human participants were in accordance with the ethical standards of the institutional and/or national research committee and with the 1964 Helsinki declaration and its later amendments or comparable ethical standards.

Open Access This article is distributed under the terms of the Creative Commons Attribution 4.0 International License (http:// creativecommons.org/licenses/by/4.0/), which permits unrestricted use, distribution, and reproduction in any medium, provided you give appropriate credit to the original author(s) and the source, provide a link to the Creative Commons license, and indicate if changes were made.

\section{References}

1. Heinberg CJ. A new synthetic anti-histaminic substance, antistine. Eye Ear Nose Throat Mon. 1947;26:639-41.

2. McKechnie JK. The cardiac action of the antihistamine compounds. S Afr Med J. 1952;26:609-13.

3. Angelakos ET. Influence of pharmacological agents on spontaneous and surgically induced hypothermic ventricular fibrillation. Ann N Y Acad Sci. 1959;80:351-64.

4. Angelakos ET, Hegnauer AH. Pharmacological agents for the control of spontaneous ventricular fibrillation under progressive hypothermia. J Pharmacol Exp Ther. 1959;127:137-45.

5. Kline SR, Dreifus LS, Watanabe Y, et al. Evaluation of the antiarrhythmic properties of antazoline. A preliminary study. Am J Cardiol. 1962;9:564-7.
6. Dreifus LS, McGarry TF, Watanabe Y. Clinical and physiological effect of antazoline, a new antiarrhythmic agent. American Heart Journal. 1963;65:607-14.

7. Shah SS, Vaidya CH, Doshi HV. Antazoline in the treatment of cardiac arrhythmias. Postgrad Med J. 1972;48:304-7.

8. Reynolds EW Jr, Baird WM, Clifford ME. A clinical trial of antazoline in the treatment of arrhythmias. Am J Cardiol. 1964;14:513-21.

9. Leon-Sotomayor L. A clinical evaluation of the antiarrhythmic properties of antazoline. Am J Cardiol. 1963;11:646-53.

10. Lanng Nielsen J, Dahl R, Kissmeyer-Nielsen F. Immune thrombocytopenia due to antazoline (Antistina). Allergy. 1981;36: 517-9.

11. Kuch M, Janiszewski M, Dluzniewski M, et al. Antazoline - ineffective or misprized in the treatment of paroxysmal atrial fibrillation? Pol J Cardiol. 2000;3:247-51.

12. Piotrowski R, Krynski T, Baran J, et al. Antazoline for rapid termination of atrial fibrillation during ablation of accessory pathways. Cardiol J. 2014;21:299-303.

13. Balsam P, Kozluk E, Peller M, et al. Antazoline for termination of atrial fibrillation during the procedure of pulmonary veins isolation. Adv Med Sci. 2015;60:231-5.

14. Farkowski MM, Maciag A, Zurawska M, et al. Comparative effectiveness and safety of antazoline-based and propafenone-based strategies for pharmacological cardioversion of short-duration atrial fibrillation in the emergency department. Pol Arch Med Wewn. 2016;126:381-7.

15. Maciag A, Farkowski MM, Chwyczko T, et al. Efficacy and safety on antazoline in the rapid conversion of paroxysmal atrial fibrillation (the AnPAF study). Europace. 2017;19:1637-42.

16. Piotrowski R, Giebultowicz J, Baran J et al. Antazoline-insights into drug-induced electrocardiographic and hemodynamic effects: Results of the ELEPHANT II substudy. Ann Noninvasive Electrocardiol 201725.

17. Guerra F, Matassini MV, Scappini L, et al. Intravenous vernakalant for the rapid conversion of recent onset atrial fibrillation: systematic review and meta-analysis. Expert Rev Cardiovasc Ther. 2014;12: 1067-75.

18. Camm AJ, Capucci A, Hohnloser SH, AVRO Investigators, et al. A randomized active-controlled study comparing the efficacy and safety of vernakalant to amiodarone in recent-onset atrial fibrillation. J Am Coll Cardiol. 2011;57:313-21. 